\title{
Esencia y relevancia de la misión para la gobernanza de la universidad privada
}

\author{
Essence and relevance of a mission statement for governance in private universities
}

\author{
LARREÁTEGUI, Carlos A. ${ }^{1}$
}

\begin{abstract}
Resumen
La misión de la universidad ha sido objeto de múltiples estudios y constituye el norte que guía estrategias y definición de metas, y traduce el sentido, la orientación, valores y principios fundacionales que orientan a estudiantes y familiares a elegir esta y no otra institución. Se propone analizar el contenido de las declaraciones de catorce universidades privadas ecuatorianas. Por medio del análisis de contenido y categorización binaria, se definieron categorías fundamentales, frecuentes o menos frecuentes en la gobernanza universitaria.

Palabras clave: declaración, misión, gobernanza universitaria
\end{abstract}

\begin{abstract}
The mission of the university has been the object of multiple studies and is the guiding element for the definition of strategies and goals. It translates the sense, orientation, values and founding principles that guide students and their families to choose this institution and not another institution. The purpose of this study was to analyze the content of the declarations of fourteen Ecuadorian private universities. Through content analysis and binary categorization, fundamental, frequent or less frequent categories were defined in university governance.
\end{abstract}

Key words: statement, mission, university governance

\section{Introducción}

La misión de la universidad ha sido objeto de numerosos estudios y pensada desde diversos contextos, lo que ha propiciado simposios, discusiones y manifestaciones de toda índole.

Ortega y Gasset (2001), sentó pautas en la conceptualización y definición de la misión de la universidad. Precisó que, la enseñanza universitaria está compuesta por dos elementos fundamentales: la formación de profesiones intelectuales y la investigación científica. Desde que escribe este Ensayo, alerta tempranamente contra los riesgos del "especialismo" (sic) y el "profesionalismo", lo que conlleva el riesgo de que una persona llegue a saber mucho de una disciplina, pero ignore prácticamente todo de todas las demás. Esta visión prospectiva marca el

\footnotetext{
${ }^{1}$ Canciller, Universidad de Las Américas, Quito, Ecuador. carlos.larreategui@udla.edu.ec
} 
compromiso de la universidad de formar profesionales integrales, para estar en mejores condiciones de dar respuesta a las necesidades de la sociedad. El autor definió tres funciones sobre las misiones de la universidad: 1) transmisión de la cultura; 2) enseñanza de las profesiones y 3) investigación científica y educación de nuevos hombres de ciencia; y la cultura como un elemento fundamental,

"... yo haría de una "Facultad" de la Cultura el núcleo de la Universidad y de toda la enseñanza superior. La cultura y las profesiones no pueden quedar encerradas y asiladas en la propia Universidad; ambas realidades deben estar en contacto con la ciencia, con la investigación, procurar contacto con laboratorios, seminarios, centros de discusión. Ese ha de ser el "humus" de la enseñanza superior... la universidad tiene que estar también abierta a la plena actualidad, más aún tiene que estar en medio de ella, sumergida en ella. Para ello la universidad tiene que intervenir en la actualidad, tratando grandes temas del día desde su punto de vista propio -cultural, profesional y científico"

La misión es el espíritu que designa la naturaleza de la institución, determina el modelo académico y sus principios, la distingue de otro tipo de actividades y de otras instituciones de educación. Su enunciado evidenciará los fines, principios y bases del proyecto institucional que guiará al organismo universitario y motivará a sus integrantes para comprometerse con su cumplimiento. La misión reflejará el medio en que se desarrolla y al que pertenece, tomando en cuenta la globalización como elemento esencial en la época contemporánea.

La misión ubica a la institución académica en su proyecto original sin estar condicionada por factores externos, políticos o de cualquier otra índole que sobrepasen su ámbito de desarrollo. Esto la legitima; le impone su investidura ética y la Auctoritas en tanto que desarrolla la naturaleza y esencia de la universidad (Grueso, 2003, pág. 94).

[...] el tema de la misión de la universidad, la entiendo como un ideal de función, la función ideal que se asigna a sí misma la universidad con relativa independencia de las demandas que de ella hacen las instituciones, el mercado y las modas sociales. Como un darse a sí misma una función que vaya más allá de las funciones que se le quieren asignar desde afuera, y al ritmo de cada cambio en el mundo social y de cada nueva mirada desde el mundo político. Y entiendo esta misión como un indicio de la clarificación de su ética, y como un comienzo del posible aporte universitario a la consolidación de una ética de lo público.

La misión es una característica singular que diferencia cada universidad. No existe un modelo único de universidad, las instituciones de educación superior tienen sus características particulares, sus misiones y una demanda educativa específica que atender. Podrían existir tantas misiones como universidades; Levy (1995) las agrupó en "olas" o tipos de universidad. No puede existir un modelo único de universidad en el país, ya que las instituciones de educación superior tienen sus características particulares, sus misiones y una demanda educativa específica que atender. Sin embargo, lejos de que estas sean dificultades difíciles de superar son, más bien, retos a superar por la universidad ecuatoriana (Larreategui, 2016).

Para formular la misión de la universidad, según su proyecto institucional e identidad se tomarán en cuenta los factores propios y de su entorno: autonomía, libertad académica, docencia, investigación titulaciones, relaciones internacionales, administración, y compromiso social, entre otros (Bermejo Muñoz, 2014)

La universidad debe contemplar factores sensibles y específicos para fijar su misión, su fin fundamental es la formación integral del ser humano, lo que genera una repercusión directa en el desarrollo de la sociedad en general y en el ser humano en particular. Al gobierno de la universidad le corresponde, fijar su misión y el cumplimiento efectivo de la misma. 
Según Drucker, la misión de la empresa debe motivar y organizar las energías humanas a fin de cumplirla. Basado en sus propuestas, la definición y declaración de la misión debe incluir al menos, dos componentes esenciales: quedar definido el producto o servicio ofrecido y el consumidor al que va destinado y debe satisfacer (Drucker P. F., 1985, págs. 79-80). Estudios sobre la misión de la universidad privada proponen la inclusión de las partes interesadas (stakeholders) compuesto por agentes internos (la comunidad universitaria, integrada por profesores, empleados, estudiantes) y agentes externos (clientes, proveedores, competidores, empresas, industrias, etc.) que de un modo u otro intercambian y alcanzan la contribución de la universidad. La inclusión de los stakeholders en la declaración de la misión de la universidad puede tener un efecto de atracción para nuevos stakeholders, que podrían mostrar interés y aproximarse a la institución, además de los que inicialmente hayan sido considerados (Bermejo Muñoz, 2014, págs. 34-35; Rey y Peña, 2011, pág. 21).

La incorporación de las partes interesadas es relevante para involucrar a la comunidad universitaria en el cumplimiento de la misión de la universidad. Según el modelo antropológico de gestión propuesto por Pérez López, las personas, ya no solo buscan y responden a incentivos, o a la satisfacción que provoca un trabajo bien hecho, sino que también buscan sentido a lo que hacen, más allá de lo que les reporta desde un punto de vista externo e interno; las personas sienten la necesidad de atender o satisfacer necesidades de otras personas e implicarse con este propósito. se convierte en un factor decisivo que les motiva y compromete a darle cumplimiento a la misión (Pérez López J. A., 1991). El grado de identificación de los miembros con la organización a la que pertenecen se convierte en el factor decisivo para su supervivencia a largo plazo (Pérez López \& Chinchilla, 1990).

La visión de Drucker, deviene realidad en la contemporaneidad. El rol de las universidades en la sociedad ha cambiado desde focalizar su actividad en la enseñanza y la investigación; a desempeñar un rol de mayor relevancia en las contribuciones económicas a la sociedad; a las actividades empresariales y al desarrollo integral en su conjunto. El cambio es alimentado por las influencias externas y el desarrollo interno de la universidad en sí misma (Clauss et al., 2018, pág. 2).

Bajo esta visión real y prospectiva, la definición de la misión ha de tener en cuenta la contribución de la producción intelectual de la universidad: la formación de profesionales de alto estándar, la investigación, la innovación, la internacionalización; que tribute a potenciar a la sociedad y a los actores que la conforman, desde las comunidades hasta las empresas e industrias, partes interesadas que incrementan sus capacidades y al mismo tiempo retroalimentan la producción y la mejora continua del capital intelectual de la universidad.

El nuevo milenio marcó una serie de transformaciones sociales, que impactaron la necesidad de establecer con precisión la declaración de objetivos, alcance e intenciones de las instituciones de educación superior, lo que significó expresar en la misión de la universidad, su responsabilidad y capacidad de rendición de cuentas a las partes interesadas.

La función de las universidades se ha modificado en correspondencia con los cambios del entorno económico, político y social. Ha pasado de la enseñanza clásica, de la creación de conocimiento y de métodos de enseñanza reproductivos; a la generación y transferencia de conocimientos a la sociedad a partir del desarrollo creativo de su capital intelectual. Los productos de la universidad no se quedan dentro de sus muros (torres de marfil), sino que les trasciende contribuyendo al desarrollo económico y social. Estas capacidades de la universidad fuera del ambiente académico transforman la misión y función institucional integral de las universidades (Rojas et al., 2018). Actualmente la misión de las universidades se ha expandido más allá de los límites de la enseñanza e investigación, para empatar con la sociedad del conocimiento (Bueno E. , 2007). 
El tránsito ha sido caracterizado desde la primera misión de las universidades,por la difusión del conocimiento como instrumento de animación individual, más que como herramienta para satisfacer las necesidades sociales; la segunda misión, por la inclusión de la investigación, lo que constituyó una revolución académica. Después de dos siglos de "decadencia", donde la universidad no fue capaz de enfrentarse a los cambios y estuvo relegada a un papel marginal; en el siglo XIX evolucionó a una nueva clase de institución; conservó rasgos de su precursora medieval, incorporó metodologías de investigación científica, desarrolladas previamente en el siglo XVIII por las sociedades académicas. Durante este periodo resurgieron las universidades como instituciones dedicadas a la producción de conocimiento y predominó la idea de una universidad orientada hacia la investigación. La inclusión de la investigación, vinculada con la reforma alemana, de principios del siglo XIX, marcó el fin de la universidad medieval y el inicio de la universidad moderna (Wittrock, 1996).

En los años 60 del siglo pasado se formuló la idea de la "tercera misión". La evolución tecnológica fue el factor determinante para que la sociedad demandara nuevas prácticas, generar e impulsar la "economía del conocimiento" a la institución universitaria. Las universidades empezaron a desarrollar la tercera misión, producir conocimiento aplicable, promover la innovación, formar y reciclar profesionales cualificados, valorizar la investigación y fomentar proyectos emprendedores o de desarrollo territorial en colaboración con el resto de agentes del sistema económico" (ACUP, Asociación Catalana d'Universitats Públiques, 2008).

El conocimiento dejó de ser un fin en sí mismo; para convertirlo en un valor de impulso a la economía desde la propia institución universitaria; sustentado en conceder a los productos intangibles un valor intelectual y económico para la sociedad, hasta ese momento ignorado en las economías. Lo que demandó formar perfiles creativos y derivar el acceso a la cultura, la ciencia y la tecnología. La universidad se ha convirtió en un elemento activo de y para la sociedad.

A finales de los años noventa se consolidó el concepto de "emprendimiento" y de "universidad emprendedora" (Clark B. R., 1998), se consideró a la institución universidad como "agente de la triple hélice: administración, academia y empresa" (Etzkowitz \& Leydesdorff, 1997). Este rol de la universidad comprometió nuevos marcos de actuación para los gobiernos de las universidades y en la definición de la misión.

Fomentar el emprendimiento y la innovación no es únicamente responsabilidad de la universidad, sino también de la sociedad; de las organizaciones, empresas e industrias, y del Estado para no limitar ni obstruir la articulación entre los diversos actores involucrados. Cualquier política y herramienta dirigida a fortalecer la tercera misión debe contar con una aproximación entre la universidad, la sociedad y la empresa, que son, al mismo tiempo, quienes demandarán los productos como resultado de su desarrollo e implementación.

Basada en esta integración, Ruiz Corbella, (2019, pág. 15) destacó la relevancia de la misión de la universidad al articular la docencia, la investigación y la transferencia de conocimientos a la sociedad. Estos resultados devienen áreas clave para la gestión de la institución,

"Está claro que la misión de la universidad debe conjugar la docencia y la transmisión de la cultura, junto con la investigación, la transferencia del conocimiento y la dimensión social de esta institución. A lo largo de las últimas décadas se ha dado más relevancia a la investigación, a la transferencia del conocimiento, a la innovación dirigida al desarrollo económico de las naciones que, a la docencia, a la dimensión social, o a la transmisión de la cultura. No es cuestión de ver si damos prioridad a una u otra misión, sino de desarrollar todas ellas de forma equilibrada y convergente; sin la relación profesor-alumno, la Universidad deja de serlo, pero sin la creación de nuevos conocimientos a través de la investigación, no es Universidad" 
Bermejo en sus estudios sobre la misión de la universidad privada, resume el enfoque dado por diversos autores, posiciones relacionadas con las organizaciones empresariales, que el autor asimila para su consideración en la definición de la misión para la universidad privada, que se resumen a continuación (Bermejo Muñoz, 2014):

Desde un primer punto de vista la misión debe estar bien escrita, clara y concisa; transmitir sentimientos y emociones, bien redactada y con buen tono, dado que afecta la imagen de la organización (Cochran, David, \& Gibson, 1985, págs. 27-39); El modelo de misión de Campbell y Nash (1992, págs. 19-27) incluye cuatro elementos: el propósito (contribución a los stakeholders y la sociedad en general; la estrategia (entendida como posicionamiento competitivo y competencias distintiva); los valores (creencias fundamentales) y los estándares y comportamiento (políticas y modos de comportamiento que sustentan el sistema de valores y competencia distintiva). Según estos autores el elemento más interesante y novedoso que se introduce es la inclusión de la estrategia y considerar que una misión fuerte se caracteriza porque cada uno de los elementos debe reforzar a los otros. Según Pearce (1982) la misión proporciona a los directivos una unidad de dirección que trasciende necesidades individuales que tienden, por su propia naturaleza, a ser estrechas y temporales, conecta con las creencias, deseos y aspiraciones de un emprendedor o fundador, consolida valores en el tiempo por encima de individuos o grupos y juega un papel crítico para evitar que posibles desviaciones y acciones a corto plazo entren en conflicto con la propuesta a largo plazo que representa.

La misión de la universidad privada constituye el eje del modelo académico y de gestión sobre la base de sus valores y principios fundacionales; marca el sentido de su puesta en marcha y funcionamiento. La misión ubica a la institución académica en su proyecto original sin estar condicionada por factores externos, políticos o de cualquier otra índole que sobrepasen su ámbito de desarrollo. Al gobierno de la universidad le corresponde, fijar su misión y el cumplimiento efectivo de la misma. Dada su importancia, el propósito de este estudio fue analizar las declaraciones de la misión en catorce universidades privadas ecuatorianas. Los resultados del estudio tienen como propósito servir de insumo para una propuesta conceptual de misión en un gobierno para la universidad privada ecuatoriana.

\section{Metodología}

Definir con claridad la misión de la universidad es fundamental. La misión constituye la referencia para el direccionamiento de estrategias, objetivos, metas, compromiso hacia la comunidad; ámbito de influencia, imagen de la institución y base de alineación de todos los procesos estructurales y organizativos: tipo de gobierno, reclutamiento de personal, formas de comunicación, integración a los procesos estratégicos y todos aquellos que son pautas para la gobernanza universitaria.

Se analizaron 14 declaraciones de misión de universidades privadas del Ecuador, con el propósito de valorar categorías y contenidos orientados al quehacer de la institución de educación superior.

En el proceso de la investigación, se analizó la construcción y declaración de la misión. Para la construcción de una matriz cualitativa de las categorías a analizar, se tomaron como base los estudios realizados por Bermejo Muñoz (2014), sustentado en las teorías de Rey Peña (2011) y el marco antropológico de la obra de Pérez López (1991) y otros autores que vincularon el cumplimiento de la misión con la motivación de las personas en la organización.

Para evaluar las categorías se asignó un valor o código binario, si la categoría está presente en la declaración de la misión se asigna uno (1), si no está presente cero (0). Asignar valores permitió analizar la frecuencia de coincidencias en los vocablos contenidos en la declaración de misión. De la interpretación derivaron componentes que sirvieron para formular un cuadro de categorías deseables para la declaración a incluir en la propuesta del modelo conceptual de gobierno universitario. 
Las bases para el análisis de la declaración de la misión de la universidad privada fueron: a) utilizar términos adecuados, conceptos claros para las partes interesadas involucradas (stakeholders); b) clara y concisa; c) tono positivo e inspirador; d) ideas distribuidas por párrafos; e) inclusión de propuestas o resultados clave; f) inclusión en frases cortas de cada uno de los stakeholders, al menos sobre estudiantes, profesores y sociedad. En el ámbito de las misiones se plantean como ejes estratégicos: la formación, la investigación y la tercera misión (innovación, emprendimiento); en el ámbito de personas los ejes estratégicos son: personal docente e investigador (PDI), estudiantes y personal de administración y servicios (PAS); en el ámbito de fortalecimiento de capacidades se plantean cuatro ejes estratégicos: financiación y gobernanza, internacionalización, evaluación (individual y colectiva) y comunicación universitaria; en el ámbito de entorno los ejes estratégicos son: universidad-ciudadterritorio, contribución socioeconómica y entornos inclusivos, sostenibles y saludables. El análisis estuvo dirigido a identificar fortalezas y debilidades y brechas formuladas según criterios de Bermejo Muñoz (2014).

\subsection{Categorías definidas en base al análisis}

\section{Características de la declaración}
a. Claridad y concisión
b. Positiva e inspiradora
c. Inclusión de áreas clave
d. Mención de los stakeholders
e. Temporalidad

\section{Misión formal (contenido en la declaración)}
a. Docencia
b. Investigación
c. Extensión
d. Calidad
e. Formación de líderes
f. Sociedad
g. Generación de conocimientos
h. Innovación
i. Ámbito nacional
j. Ámbito internacional

3. Áreas clave por partes interesadas (stakeholders)
a. Formación integral (Estudiantes)
b. Desarrollo docente-investigativo (Profesores)
c. Personal de Administración y Servicios (PAS)
d. Formación avance profesional (Sociedad)
e. Transferencia de conocimientos y tecnología (Sociedad)
f. Responsabilidad social (Sociedad)
g. Retorno económico para desarrollo propuesta específica (Promotores) 


\section{Resultados y discusión}

Del análisis de las declaraciones de misión de las catorce universidades privadas ecuatorianas estudiadas se identificaron debilidades, fortalezas y barreras en el contenido de las mismas, que permitieron aportar elementos para la propuesta conceptual de la misión de un modelo de gobierno de la universidad privada.

La palabra misión no estuvo incluida en ninguna de las declaraciones, por su contenido dejan claro el "para qué" existe la institución de educación superior, se sobreentiende por sí sola, y es suficiente.

Los resultados del análisis cuantitativo fueron: las 14 universidades estudiadas tienen una declaración formal explícita. El promedio de palabras utilizadas en la declaración fue de 51, en un intervalo de mínimo de palabras (21) correspondiente a la Universidad San Francisco de Quito, y un máximo de palabras (224), en la declaración de la Universidad Casa Grande. Se debe considerar que la propuesta por algunos autores, de inclusión de las partes interesadas y su participación en la declaración de la misión, cambia el criterio de extensión. En tal caso la redacción de la misión debe incluir como mínimo, la intención/ especificidad de la universidad y el usuario/s, a lo que se añadirían otras partes interesadas. Es un tema que queda a discreción del gobierno de cada universidad.

Universidad San Francisco de Quito

"La USFQ busca formar individuos librepensadores, innovadores, creativos, emprendedores en el marco de las Artes Liberales y bajo sus principios fundacionales".

Universidad Casa Grande

"Formar personas éticas y socialmente responsables, proactivas, innovadoras, reflexivas, con capacidad investigativa y comprometidas con su profesión para el desarrollo de la sociedad desde las disciplinas humanas y sociales; que generen nuevos emprendimientos y conocimientos, propiciando un contexto incluyente, intercultural, diverso y de equidad de género. Para hacer realidad su misión, la Universidad Casa Grande prioriza el:

- Formar seres humanos integrales, que se caractericen por su eficiencia profesional y su creatividad en el ámbito de las ciencias humanas y sociales.

- Trabajar en red con diversos países y culturas, con la tecnología necesaria para el máximo aprovechamiento de las posibilidades humanas, pero considerando el perfil de cada persona, de modo que se consolide como una fuente de propuesta de aprendizaje desde América Latina.

Contar con pedagogías innovadoras para aprender a:

- Crear, aprender haciendo, aprender a reflexionar durante y después de la acción, y, por lo tanto, aprender a ver el mundo desde una óptica que permita generar y asimilar cambios en sus diferentes áreas de desempeño.

- Desarrollar su tarea en un marco de rigor académico, que enfatice la importancia de la investigación y la educación continua; de exposición e interacción creativa con el estado del arte en las ciencias humanas y sociales; pluralista y ético, de respeto a la persona humana y a su diversidad cultural, que fomente la equidad y el desarrollo humano sostenible".

El análisis cuantitativo del contenido de las misiones estudiadas según las categorías propuestas: exposición clara, concisa, positiva e inspiradoras (93\%). Evaluar el criterio de la declaración como "inspiradora" fue complejo. El significado del concepto involucra el atraer o sugerir elementos que conduzcan a que el otro, el cliente, las partes interesadas, los promotores demanden su uso, o el proveedor se interese en el mismo. La 
esencia de la actividad de una universidad es la formación del capital humano y la transferencia de conocimientos, y para que sea inspiradora la misión tiene que contener elementos sustantivos de atracción, de creación y desarrollo individual o colectivo que conduzca a que prefiere esa universidad y no otra. En las declaraciones de las universidades el "concepto" inspirador estuvo pendiente (Tabla 1).

Tabla 1

Características de la declaración

\begin{tabular}{|c|c|c|c|c|c|c|}
\hline \multirow[t]{2}{*}{ UNIVERSIDAD } & \multicolumn{6}{|c|}{ CATEGORÍAS } \\
\hline & $\begin{array}{l}\text { No. } \\
\text { palabras }\end{array}$ & $\begin{array}{l}\text { Claridad } \\
\text { Concisión }\end{array}$ & $\begin{array}{l}\text { Positiva } \\
\text { Inspiradora }\end{array}$ & $\begin{array}{l}\text { Áreas } \\
\text { Clave }\end{array}$ & $\begin{array}{l}\text { Mención } \\
\text { stakeholders }\end{array}$ & Temporalidad \\
\hline $\begin{array}{l}\text { Universidad tecnológica } \\
\text { empresarial de Guayaquil }\end{array}$ & 36 & 1 & 1 & 0 & 0 & 0 \\
\hline $\begin{array}{l}\text { Universidad particular } \\
\text { internacional SEK }\end{array}$ & 28 & 1 & 0 & 0 & 0 & 0 \\
\hline Universidad Casa Grande & 224 & 1 & 1 & 0 & 0 & 0 \\
\hline Universidad del Río & 97 & 0 & 1 & 1 & 1 & 0 \\
\hline $\begin{array}{l}\text { Universidad Tecnológica - } \\
\text { ECOTEC }\end{array}$ & 27 & 1 & 1 & 1 & 0 & 0 \\
\hline $\begin{array}{c}\text { Universidad Regional autónoma } \\
\text { de los Andes - UNIANDES }\end{array}$ & 47 & 1 & 1 & 0 & 0 & 0 \\
\hline Universidad Israel & 32 & 1 & 1 & 0 & 0 & 0 \\
\hline $\begin{array}{l}\text { Universidad San Francisco de } \\
\text { Quito }\end{array}$ & 21 & 1 & 1 & 0 & 0 & 0 \\
\hline $\begin{array}{l}\text { Universidad Tecnológica } \\
\text { Indoamérica }\end{array}$ & 32 & 1 & 1 & 0 & 0 & 0 \\
\hline $\begin{array}{l}\text { Universidad de Especialidades } \\
\text { Espíritu Santo }\end{array}$ & 31 & 1 & 1 & 0 & 0 & 0 \\
\hline Universidad Otavalo & 58 & 1 & 1 & 0 & 0 & 0 \\
\hline $\begin{array}{l}\text { Universidad internacional del } \\
\text { ecuador- UIDE }\end{array}$ & 9 & 1 & 1 & 0 & 0 & 0 \\
\hline Universidad de los Hemisferios & 40 & 1 & 1 & 0 & 0 & 0 \\
\hline $\begin{array}{c}\text { Universidad Particular San } \\
\text { Gregorio }\end{array}$ & 36 & 1 & 1 & 0 & 0 & 0 \\
\hline Media & 51 & & & & & \\
\hline $\begin{array}{l}\text { Universidad tecnológica } \\
\text { empresarial de Guayaquil }\end{array}$ & 36 & 1 & 1 & 0 & 0 & 0 \\
\hline \multirow[t]{3}{*}{ Media } & 51 & & & & & \\
\hline & & 13 & 13 & 2 & 1 & 0 \\
\hline & $\%$ & 93 & 93 & 14 & 7 & 0 \\
\hline
\end{tabular}

Las declaraciones fueron formales para conminar la selección del producto en el segmento de población objeto; usuarios: estudiantes y padres; proveedores e incluso inversores conminados al desarrollo de instituciones de educación superior. De tal modo es relevante la importancia de cómo se construye, se determinan los contenidos y se redacta la misión.

En ninguna de las declaraciones de misión se especificó la temporalidad. Este carácter no se considera imprescindible. Según los criterios de Rey Peña (2011, pág. 40), la misión de una empresa no debería cambiar 
mientras no existan causas que lo justifiquen (venta, cambios significativos del entorno, internacionalización); lo que se considera asimilable para las universidades. Se debe tener en cuenta, que la "misión" acorde con un análisis político y estratégico de la universidad, puede mejorarse, modificarse, cuando responda a su propio desarrollo.

Rara vez una misión puede tener una esperanza de vida mayor a 30 o como mucho 50 años, y, por lo general, la validez que podría esperarse está en torno a los 10 años. De lo que trata, es como se construye la misión de la universidad, con la participación de todas las partes interesadas, Rey Peña (2011, pág. 89) citando a Drucker (Drucker P. , 1974). Este criterio se debe tener en cuenta en la propuesta de un modelo conceptual de gobierno para la universidad.

Con referencia a los contenidos de la misión, su tipo y alcance, el análisis de los datos se realizó en función de la aparición o no del vocablo específico que, permitió entender la inclusión de la función en la universidad. No siempre el contenido del vocablo fue exacto, cuando el contenido respondió al criterio de análisis, se decidió un criterio "proxi" para la interpretación y calificación, con el propósito de minimizar la dispersión del dato; tal es el caso del criterio calidad/excelencia, o la decisión de agrupar, por su sentido, sociedad/desarrollo/sostenibilidad/ diversidad; los otros criterios fueron generación de conocimientos, innovación/emprendimiento; ámbito nacional (refirió relaciones con sectores o influencia a nivel del país) y el ámbito internacional cuando específico términos asociados con la internacionalización.

El comportamiento fue como sigue, el $79 \%$ de las declaraciones incluyó la docencia (formación); $57 \%$ la investigación, ninguna declaración incluyó la extensión universitaria como parte de sus procesos, que integra la comunidad universitaria a su entorno social. Esto reflejó que el centro de actividad universitaria no está orientada a la investigación, a la generación y transferencia de conocimientos; ni la extensión universitaria es objeto esencial de la gestión de la universidad.

El 57\% de las declaraciones incluyeron los vocablos de calidad/ excelencia y sólo un 14\% la formación de líderes. Expresar la formación de líderes en la misión, es un elemento motivador para acceder a la universidad porque lleva implícito la capacidad de la institución a desarrollar actitudes en estudiantes y futuros profesionales, en fomentar principios y valores en el desarrollo de la profesión, da seguridad y confianza para entrar en la sociedad, en el mercado de trabajo con mejores habilidades y destrezas (Escudero, 2020).

El impacto en la sociedad, es un elemento fundamental en el desempeño de la universidad contemporánea; el 71\% declaró vocablos asociados a este concepto: sociedad, desarrollo, sostenibilidad o diversidad; el $43 \%$ la generación de conocimientos; $50 \%$ la innovación/emprendimiento; $71 \%$ influencia o relación con el ámbito nacional de influencia y solo un $21 \%$ abordó el tema de la internacionalización de la universidad. La internacionalización, debe ser un área clave, es un proceso complejo de carácter dual, implica la presencia de la dimensión internacional al interior de sus funciones, y por otra, implica la proyección internacional de sus capacidades y productos. Ofrece oportunidades, para la mejora de la calidad de la docencia y la investigación; la vinculación, articulación y fortalecimiento institucional por lo que debe ser declarada como parte visible del desarrollo institucional (Sebastián, 2005).

Con referencia a las áreas clave de resultados, en la Tabla 2 se describen las áreas clave en correspondencia con las partes interesadas que fueron consideradas en el estudio. 
Tabla 2

Partes interesadas (stakeholders) y Áreas clave de la misión

\begin{tabular}{|c|c|}
\hline Stakeholders (Partes interesadas) & Áreas clave de la misión \\
\hline Estudiantes & Formación integral \\
\hline $\begin{array}{c}\text { Profesores y Personal de Administración y } \\
\text { Servicios (PAS) }\end{array}$ & $\begin{array}{c}\text { Desarrollo docente e investigador } \\
\text { Desarrollo profesional }\end{array}$ \\
\hline Sociedad & $\begin{array}{c}\text { Formación de profesionales, avance y } \\
\text { transferencia de conocimientos }\end{array}$ \\
\hline Promotores & $\begin{array}{c}\text { Retorno económico para desarrollo de la } \\
\text { propuesta específica }\end{array}$ \\
\hline
\end{tabular}

El 71\% de las declaraciones incluyeron la formación integral dirigida a los estudiantes, ninguna declaración expresó acciones de desarrollo para el personal docente, investigador o profesional, ni al personal de administración y servicios; el $7 \%$ declaró la formación y avance profesional en su impacto en la sociedad; un 29\% la transferencia de conocimientos y la responsabilidad social de la universidad; en ningún caso se consideró el retorno económico para desarrollo de la propuesta específica.

En resumen, la variabilidad en la declaración de la misión de las diferentes universidades permitió sistematizar criterios, y evaluar las categorías recomendables a incorporar en una propuesta conceptual de misión para la gestión de un gobierno universitario de la universidad privada.

A partir de los resultados de la investigación, en la declaración de la misión es recomendable tener en cuenta: La participación de las partes interesadas internas y externas, una construcción participativa de abajo arriba conducida por los líderes de la universidad, basada en sus principios fundacionales; El conjunto de ideas que se aporten incluirá las mejores criterios, categorías o variables que expresen la intención e identidad de la universidad; y el compromiso y motivación de las personas que participaron en su construcción en correspondencia con el modelo antropológico de gestión de Pérez López (1991).

Categorías a tener en cuenta en la construcción de la misión. La declaración explícita corresponderá a los criterios definidos por el gobierno de la universidad.

a. Áreas clave de resultados, descripción de los elementos o variables que la componen,

b. Partes interesadas por áreas clave de resultados

c. Objetivos a seguir para cada área de resultado, definir temporalidad para su cumplimiento.

d. Medidas para evaluar el avance, que sirvan de base para la toma de decisiones y mejora continua,

e. Diseño de indicadores en función de las medidas de evaluación (Rey Peña (2011, pág. 169) propone un cuadro de mando integral orientado a la misión, asimilable a la gestión universitaria)

Sistema de monitoreo o auditoría interna para el seguimiento de los indicadores orientados a la misión.

\section{Conclusiones}

La construcción de la misión en la universidad privada debe caracterizarse por ser un proceso participativo de abajo a arriba; que tenga en cuenta las necesidades de la sociedad y del capital humano que conforma los estamentos universitarios, de modo que su involucramiento en el proceso de elaboración de la misión sea parte 
de su motivación; motivación trascendente de la recepción de los logros, y de la contribución personal y colectiva que se da a la organización; todo ello es parte impulsora de su cumplimiento.

En el modelo de gobierno, la declaración de la misión es el eje conductor. La participación comprometida de la comunidad que conoce los objetivos, las áreas clave de resultados asociadas a los diferentes niveles estructurales de la universidad, conmina a la participación y aporte personal comprometido. El conocimiento y participación colectiva, permite que los miembros de la organización, independientemente del nivel, función o tarea que desempeñen, conozcan y tengan bien definida su contribución a la misión.

La inclusión en la declaración de la misión de las partes interesadas, constituye un elemento esencial para la definición de la estructura de gobierno y los procesos de gobernanza. Son los actores involucrados externos e internos de la universidad los que juegan un papel fundamental en la generación, producción y transferencia del conocimiento. La implantación efectiva de una misión en una institución universitaria solo será posible, si los miembros de la institución son conscientes de su existencia y se movilizan hacia ella mediante el impulso de una motivación consistente.

\section{Referencias}

ACUP, Asociación Catalana d'Universitats Públiques. (2008). Libro Blanco de la Universidad de Cataluña. Barcelona, España: Universidad de Cataluña.

Bermejo Muñoz, L. (2014). La misión en el gobierno de instituciones universitarias de iniciativa privada. Recuperado el 15 de mayo de 2020, de http://www.tdx.cat/handle/10803/368200

Bueno, E. (2007). La tercera misión de la universidad: El reto de la transferencia del conocimiento. Revista de Investigación en Gestión de la Innovación y Tecnología (41), 2.

Clark, B. R. (1998). Creating entrepreneurial universities: Organizational pathways of transformation. New York: Oxford.

Clauss, T., Moussa, A., \& Kesting, T. (2018). Entrepreneurial University: A stakeholder-based conceptualization of the current state and an agenda for future research. International Journal of Technology Management, 77(1/2/3), 109-144. https://doi.org/10.1504/ijtm.2018.091726

Drucker, P. (1974). Management: tasks, responsibilities, practices. New York: Harper \& Row.

Drucker, P. F. (1985). Management: Tasks, Responsibilities, Practices. New York: Harper Business.

Escudero, F. (2020). Importancia del liderazgo en la vida universitaria. Recuperado el 20 de 10 de 2020, de https://silfersystem.com/index.php/15-novedades/articulo/25-importancia-del-liderazgo-en-la-vidauniversitaria

Etzkowitz, H., \& Leydesdorff, L. (1997). Introduction to special issue on science policy dimensions of the Triple Helix of university-industry-government relations. https://doi.org/10.1177/095042229801200402

Grueso, D. I. (2003). Universidad y ética de lo público: Reflexiones para un plan de desarrollo. Pensamiento Universitario, 1, 91-106.

Larreategui, C. (2016). Diálogo entre rectores. Universidad del Futuro. Pontificia Universidad Católica del Ecuador. Quito, Ecuador. 
Levy, D. (1995). La educación superior y el estado en Latinoamérica: Desafíos privados al predominio público. México: CESU / FLACSO / Miguel Angel Porrúa.

Ortega y Gasset, J. (2001). Misión de la universidad. Madrid: Alianza Editorial.

Pearce, J. (1982). The Company Mission as a Strategic Tool. Sloan Management Review, 23(3), 15-24.

Pérez López, J. A. (1991). Teoría de la acción humana en las organizaciones. Madrid: Rialp.

Pérez López, J., \& Chinchilla, N. (1990). ¿Empresa o negocio?: Distintos enfoques para la dirección de personas en las organizaciones. División de Investigación del IESE, FHN-161. Barcelona.

Rey Peña, C. (2011). La misión de la empresa. Barcelona, España: Programa de Doctorado. Universidad Internacional de Catalunya.

Rojas, M. D., Canal Carrillo, A. I., \& Córdova Félix, J. (2018). La tercera misión de la universidad: Evolución y diversas actividades. XIII Congreso Internacional de Contaduría, Administración e Informática. Ciudad Universitaria, México.

Ruiz-Corbella, M., \& López-Gómez, E. (2019). La misión de la universidad en el siglo XXI: comprender su origen para enfrentar el futuro. Revista de la Educación Superior, 48(189), 1/19.

Sebastián, J. (2005). La internacionalización de las universidades como estrategia para el desarrollo institucional. Innovación Educativa, 5(26). Recuperado el 20 de octubre de 2020, de https://www.redalyc.org/articulo.oa?id=1794/179421475009

Wittrock, B. (1996). Las tres transformaciones de la universidad moderna,. En S. Rothblatt y B. Wittrock (Eds.), La universidad europea y americana desde 1800: las tres transformaciones de la universidad moderna, (pp. 331-394). Barcelona, España: Ediciones Pomares-Corredor. 